\title{
A Review on Adverse Drug Events to Create Awareness
}

\section{Pallavi Jaiswal}

School of Bio Sciences and Technology, VIT University, Vellore, India

*Corresponding author: Pallavi Jaiswal, School of Bio Sciences and Technology, VIT University, Vellore, India, E-mail: jmd.pallavi@gmail.com

Received date: May 12, 2014, Accepted date: May 29, 2014, Published date: June 6, 2014

Copyright: (c) 2014 Jaiswal. This is an open-access article distributed under the terms of the Creative Commons Attribution License, which permits unrestricted use, distribution, and reproduction in any medium, provided the original author and source are credited.

\begin{abstract}
Adverse Drug Events (ADE) reporting is the foundation of any Pharmacovigilance system. There is always a risk in the proactive management of newly marketed drugs. Most drugs are used in a larger population after their commercialization than in clinical trials. It is important to detect the full safety profile of the drug before its marketing and throughout its marketing period. The post marketing pharmacovigilance provides the assurance of rational and safe use of medications. The medical practitioners and the pharmacovigilance body under the guidance of World Health Organization are responsible for the risk management system of a drug in different countries. The level of education and training on ADE reporting has improved the Pharmacovigilance of many drugs. Education and training is the most recognized means of improving $A D E$ reporting.
\end{abstract}

Keywords: Pharmacovigilance; Adverse Drug Events (ADE) reporting; Clinical trials; WHO

\section{Introduction}

According to the World Health Organization, Pharmacovigilance is defined as "the study of activities related to the detection, assessment, understanding and prevention of adverse effects or any other drugrelated problem" [1]. The International Drug Monitoring Programme was established after the thalidomide disaster detected in 1961 by WHO [2]. Since then 134 countries have become a part of the WHO Pharmacovigilance Programme by 2010 [1]. By its effective assessment of the risk-benefit profile of medicines and balanced information, WHO PV programme has supported public health.

The MED WATCH program of Food and Drug Administartion (FDA) in USA collects reports from medical practitioners and health care professionals about the occurrence of adverse drug events as a voluntary support [3]. The new MedWatch training program, MedWatch Learn, released by FDA teaches health professionals, students, and patients about the guidelines to complete the online FDA forms necessary to report medication errors [4].

\section{The Need of ADE Reporting}

The Adverse Drug Events has emerged as one of the major concern of world. It not only affects the adults but the children and infants age group too. Because of the variation in its rate of affecting the consumer mass, a proper assessment is required to ensure drugs safety and efficacy.

The knowledge and awareness about ADE is not enough, experience of proper assessment and reporting of $\mathrm{ADE}$ is required. The ADE reporting is done in two cases, when the reaction is serious $(77,77.8 \%)$ and unusual $(70,70.7 \%)[5,6]$. In the study conducted by Al-Hazmi, $53.24 \%$ of surveyed community pharmacists stated that ADE reporting purpose is to identify safe drugs, $29 \%$ agreed that it is useful for calculating the rate of incidence of $\mathrm{ADE}, 6 \%$ the system served as a source of information about the characteristics of ADRs, $12 \%$ of community pharmacists considered the purpose of the ADE reporting was to detect potential ADRs and whereas, $6 \%$ of surveyed community the purpose of $\mathrm{ADE}$ reporting system is to identify $\mathrm{ADE}$ within the same pharmaceutical class (Figure 1) [7].

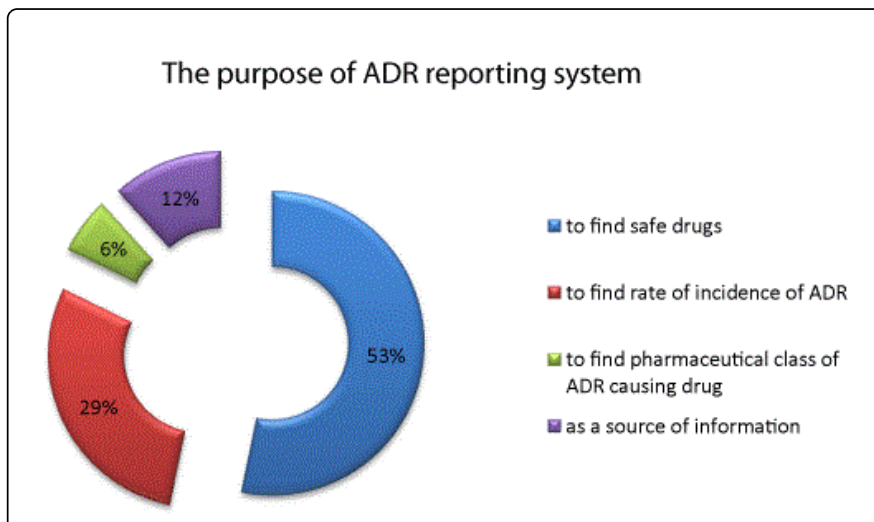

Figure 1: The purpose of ADE reporting described by Al-Hazmi et al. [7].

Inman has broadly classified factors associated with ADE underreporting among health professionals as 'seven deadly sins'. According to his description sins are caused due to the attitudes of professionals towards their activities, lack of knowledge related to the problems associated with ADE reporting and the disinterest shown by professionals [8]. This includes [5,8]:

- Financial incentives: reporting rewards.

- Legal aspects: fear of getting involved into legal costs.

- Complacency: the belief that before the commercialization, ADE reporting is well documented for a drug.

- Diffidence: the belief that a particular drug was certainly involved in the adverse drug event.

- Indifference: the belief that a single case observed by an individual doctor might not contribute to medical knowledge. 
Page 2 of 2

- Ignorance: the belief that only serious reports or unexpected ADE reporting is required.

- Lethargy: lack of interest or time to report ADE and other excuses.

\section{Role of Pharmacovigilance}

Despite being great resources, there is limited access for public to the global safety databases which allows the pharmaceutical companies to stand by their drugs and tend to blame the consumers. Therefore, there is a critical need for a comprehensive information database [2,9]. Apart from ADE reporting, pharmacovigilance bodies' helps in monitoring standard medicine factors, medication errors, efficacy reports, acute and chronic poisoning, drug-induced illness, and other key concerns required for measuring drug safety and its efficacy [10].

A study by Abideen et al. showed that the implementation of selfreporting pharmacovigilance has helped in decision making to improve patient care oriented program by the health care professionals [11]. The combination of Pharmacovigilance with other techniques, such as, high-throughput screening and virtual screening has become a standard process to utilize resources for the discovery of new drugs $[12,13]$. According to Raschi et al. pharmacovigilance analysis stated that the appropriate prescription of drugs and its dosage is vital and should be based on the underlying disease, patient's risk factors, concomitant drugs and local pattern of drug resistance [15].

\section{Conclusion}

To improve drugs safety and its efficacy it is essential for the faculty and the students to gain awareness, and experience in recognizing adverse drug reactions. It will help them in knowing the importance of participation in the national monitoring event program. This can be implemented by pharmacy schools through a course which provides information about adverse drug reaction and medication error reporting in its curricula.

The consumers can also contribute in the better understanding of the medicines safety. Through proper guidance and information about the reporting system will be resourceful to provide more data on the efficacy and safety of a drug. They should be careful about the drugs prescribed by health professionals and its dosage.

\section{References}

1. http://www.who.int/medicines/areas/quality_safety/safety_efficacy/ pharmvigi/en/

2. Dave VS (2013) Current Trends in Pharmacovigilance. J Pharmacovigilance 1:e104.

3. http://www.fda.gov/Safety/MedWatch/default.htm

4. http://www.accessdata.fda.gov/scripts/MedWatchLearn/

5. Oshikoya KA, Awobusuyi JO (2009) Perceptions of doctors to adverse drug reaction reporting in a teaching hospital in Lagos, Nigeria. BMC Clinical Pharmacology 9: 14.

6. Generali JA (2014) Adverse Drug Event Reporting: Awareness Is Not Enough. Hosp Pharm 49: 110-111.

7. Al-Hazmi NN (2013) A Study of Community Pharmacists' Awareness and Contributions to Adverse Drug Reactions (ADRs) Reporting Systems in the Makkah, Kingdom of Saudi Arabia (KSA). J Clin Trials 3: 127.

8. Inman WH (1996) Attitudes to adverse drug-reaction reporting. Br J Clin Pharmacol 41:433-435.

9. Persaud-Sharma V, Zhou SF (2013) Incorporation of New Technologies into Global Pharmacovigilance. J Pharmacovigilance 1: e102.

10. Lu Z (2009) Information technology in pharmacovigilance: Benefits, challenges, and future directions from industry perspectives. Drug Healthc Patient Saf 1: 35-45.

11. Abideen PS, Chandrasekaran K, Uma Maheswaran, Vijayakumar A, Kalaiselvan V, et al. (2013) Implementation of Self Reporting Pharmacovigilance in Anti Tubercular Therapy Using Knowledge Based Approach. J Pharmacovigilance 1:101.

12. Flower DR (2013) Pharmacovigilance, Drug Repositioning, and Virtual Screening. J Pharmacovigilance 1:e103.

13. Hattotuwagama CK, Davies MN, Flower DR (2006) Receptor-ligand binding sites and virtual screening. Curr Med Chem 13: 1283-1304.

14. Raschi E, Poluzzi E, Koci A, Moretti U, Sturkenboom M, et al. (2013) Macrolides and Torsadogenic Risk: Emerging Issues from the FDA Pharmacovigilance Database. J Pharmacovigilance 1:104.

15. Justo D, Zeltser D (2006) Torsades de pointes induced by antibiotics. Eur J Intern Med 17: 254-259. 